\title{
Biometría del Musculo Tríceps Braquial y de sus Puntos Motores. Un Estudio Anatómico Directo en una Muestra de Población Colombiana
}

\author{
Biometry of the Brachial Triceps Muscle and its Motor Points. \\ A Direct Anatomical Study in a Sample of Colombian Population
}

Edgar Adrián Torres' Luis Ernesto Ballesteros² \& Pedro Luis Forero ${ }^{3}$

TORRES, E. A.; BALLESTEROS, L. E. \& FORERO, P. L. Biometría del músculo tríceps braquial y de sus puntos motores. Un estudio anatómico directo en una muestra de población Colombiana. Int. J. Morphol., 36(3):948-954, 2018.

RESUMEN: Las características anatómicas del músculo tríceps braquial (MTB) determinan la velocidad de reparación y capacidad de generación de torque. El MTB es inervado típicamente por el nervio radial (NR), pero existen reportes de inervación por parte del nervio axilar. El propósito de este estudio fue evaluar los componentes musculotendinosos y nerviosos del MTB. Mediante disección directa en una muestra de 48 brazos de población mestiza colombiana, se evaluó morfometría musculotendinosa, ramos nerviosos, puntos motores (PM) y origen de la inervación del MTB. Las longitudes de las cabezas medial, lateral y larga del MTB correspondieron al 77,3\%,86 \% y $97 \%$ respectivamente de la longitud del brazo. El MTB fue inervado en la totalidad de la muestra por el NR quien emitió entre dos y cinco ramos motores. El primer ramo inervó la cabeza larga en el $100 \%$ de los casos y emergió lateral a la banda tendinosa de inserción del músculo latísimo del dorso. La cabeza larga recibió con mayor frecuencia tres PM (26,6 \%), ubicados en el $85 \%$ de los especímenes a 4-10 cm del origen muscular; mientras que la cabeza lateral recibió tres PM en el 44,4 \% de la muestra. No hubo diferencias estadísticamente significativas entre el número de PM del MTB izquierdo con relación al derecho (p=0,578). El área de dispersión de PM, así como la morfometría del MTB, son de relevancia clínica, debido a su uso en la estimulación eléctrica neuromuscular y transferencias nerviosas. Los resultados presentados en el presente estudio se constituyen en guía que facilita realizar estas acciones terapéuticas.

PALABRAS CLAVE: Músculo tríceps braquial; Puntos motores; Morfometría; Nervio radial.

INTRODUCCIÓN

El músculo tríceps braquial (MTB), prominente masa muscular formada por los vientres lateral, largo y medial, se ubica en el compartimento posterior del brazo. Su cabeza larga se origina en el tubérculo infraglenoideo de la escápula, mientras que las cabezas lateral y medial se originan en la superficie posterior de la diáfisis humeral, al nivel superior e inferior respectivamente del surco del nervio radial (NR). Los tres vientres confluyen en el potente tendón que se inserta en el olecranon de la ulna y en la fascia del antebrazo. El MTB es inervado por el NR, quien emite ramos al nivel axilar y del tercio proximal del brazo.(Fabrizio \& Clemente, 1997; Tubbs et al., 2006; Windisch et al., 2006).

La extensión del codo se explica por la acción sinergista del MTB y del ancóneo, con mayor contribución del primero. En la extensión del codo es la cabeza lateral la que imprime mayor potencia al movimiento en contra de resistencia. Por su parte, la cabeza larga, además de participar en el movimiento de extensión del codo, evita el desplazamiento inferior de la cabeza humeral (Windisch et al.). La presencia de una cuarta cabeza del MTB al igual que otras variaciones de esta estructura muscular, han sido señaladas especialmente en reporte de casos (Fabrizio \& Clemente; Tubbs et al.; Cheema \& Singla, 2011). Otros autores (Nayak et al., 2008; Sawant, 2013) reportan con una prevalencia del 2-4\% presencia de cuarta cabeza del MTB.

El patrón de distribución de la inervación del MTB, así como la biometría de los puntos motores (PM) para el mismo músculo ha sido pobremente estudiado. El PM anatómico se define como el punto visible en el que un ramo motor (o una de sus ramificaciones) ingresa al vientre mus-

\footnotetext{
${ }^{1}$ Fisioterapeuta, MSc. Ciencias Básicas Biomédicas, Universidad Autónoma de Bucaramanga, Colombia.

${ }^{2}$ Médico, MSc. Morfología Humana, Universidad Industrial de Santander, Colombia.

${ }^{3}$ Médico, Especialista en Patología, Universidad Industrial de Santander, Forense Instituto de Medicina Legal y Ciencias Forenses, Colombia.
} 
cular, con el fin de brindarle inervación; esto implica que la ubicación del PM determina la zona de unión neuromuscular, que determina la transmisión de la orden motora sobre el músculo estriado esquelético (Lee et al., 2009; Gobbo et al., 2011). Algunos estudios (Bertelli et al., 2007; Uerpairojkit et al., 2013; Al-Meshal \& Gilbert, 2013) concuerdan en que la inervación proviene en el 100 $\%$ de los casos del NR y el primer ramo motor (evidenciando entre uno y cinco ramos en su estudio) siempre se dirige a la cabeza larga.

El adecuado conocimiento sobre la configuración anatómica, sus patrones de inervación y PM relacionados del MTB permite aplicaciones clínico - quirúrgicas, en rehabilitación y en el uso de herramientas diagnósticas como la electromiografía (Gobbo et al., 2011; Okuma et al., 2013; Wang \& Weiss, 2013). Por tal razón, el presente estudio pretendió evaluar la expresión morfológica y biométrica tendomuscular y de los PM del MTB en una muestra de especímenes cadavéricos frescos de un grupo poblacional mestizo colombiano.

\section{MATERIAL Y MÉTODO}

Se evaluaron mediante técnica de disección convencional, 48 brazos provenientes de especímenes cadavéricos frescos no reclamados del Instituto de Medicina Legal y cadáveres preparados bajo técnica de conservación con formaldehído, sin previa intervención de la región braquial, de laboratorios de Morfología de Bucaramanga, Colombia. Se incluyeron los miembros superiores de individuos mayores de 20 años, sin signos de trauma reciente, cicatrizal o de amputación parcial del segmento.

Con el espécimen ubicado en decúbito prono, con flexión y abducción de hombro a $90^{\circ}$ y flexión de codo a $90^{\circ}$, se realizó incisión mediana posterior comprendida desde el ángulo supero-lateral de la escapula hasta el segmento inferior del olecranon, que comprometió piel, tejido celular subcutáneo y fascia del brazo. Luego, se reflejaron los colgajos fascio-cutáneos hacia lateral y medial con el fin de exponer los estructuras tendomusculares y nerviosas de la región (Fig. 1). Se registró la distribución de las ramas del NR en las cabezas del MTB, las características anatómicas y biométricas de los vientres musculares y de sus respectivos PM; Las variables morfo-métricas se evaluaron con calibrador digital (Mitutoyo $\left.{ }^{\circledR}\right)$. De igual manera, se midió la longitud del brazo tomando como punto referente proximal el porción lateral del acromion y al epicondilo lateral como límite inferior de este segmento del miembro superior.

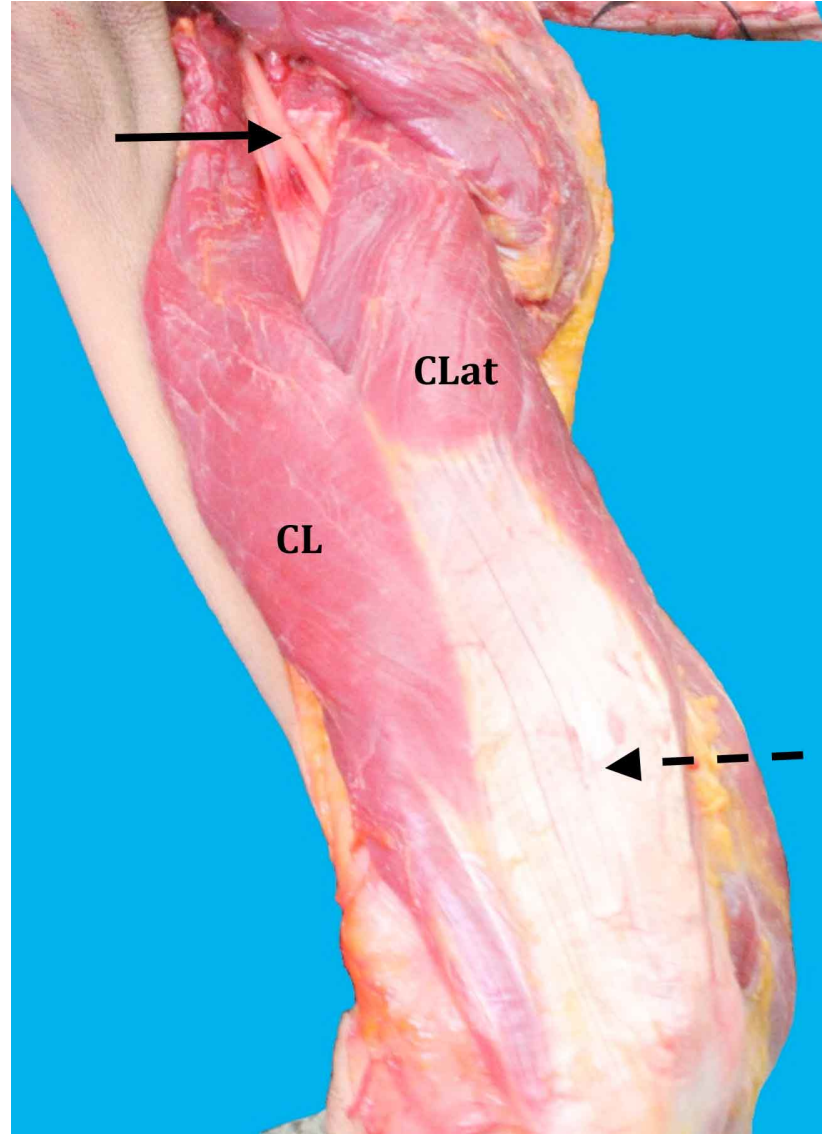

Fig. 1. Disección inicial del MTB en un brazo derecho. Se retiraron los colgajos fasciocutáneos. La línea continua señala el nervio radial; la línea discontinua la porción miotendinosa. CL: cabeza larga. CLat: cabeza lateral.

Para la medición de la longitud de las cabezas musculares del MTB y la ubicación de sus PM se tomó como referencia el punto más alto del origen muscular. Para cada una de las cabezas se midió la longitud total, longitud miotendinosa proximal y distal, distancia desde el origen muscular hasta la ubicación de cada PM, dispersión de PM, comprendida entre el primero y el más distal PM que ingresa a un vientre muscular. Además, se determinó el y punto de origen de los ramos motores del NR.

La base de datos se digitó en Excel y los análisis estadísticos se realizaron en STATA 12.0. El plan de análisis incluyó estadísticos descriptivos, aplicación de pruebas de normalidad (Shapiro-Wilk), comparaciones entre medias y medianas con t de Student y prueba de Wilcoxon, y pruebas de correlación entre variables con $r$ de Pearson \& Rho de Spearman, aceptando un nivel de significancia estadística con un valor $\mathrm{p}<0.05$ (Carter et al., 2011). El estudio contó con la aprobación del Comité de Ética de la Universidad Industrial de Santander. 


\section{RESULTADOS}

El origen de cada una de las cabezas del MTB, así como su inserción distal, presentó distribución usual en la totalidad de la muestra. No se presentó casos de cabeza adicional u otras variaciones anatómicas. La longitud del brazo fue $297,8 \mathrm{~mm}$ DE $16,6 \mathrm{~mm}$, sin diferencias significativas entre hemicuerpos $(\mathrm{P}=0,086)$.

La longitud de la cabeza medial, la más corta de las tres, representa el 79,8\% de la longitud total de la cabeza larga MTB; a su vez, la longitud de la cabeza lateral representa el 88,6 \% de la cabeza larga. No existió diferencia estadísticamente significativa entre las longitudes de las cabezas del MTB con relación al lado $(\mathrm{P}=0,159)$. El segmento miotendinoso distal presento una longitud estadísticamente mayor con relación al segmento proximal $(\mathrm{P}=0,0001)$. Las longitudes de las cabezas medial, lateral y larga correspondieron respectivamente al 77,3\%, 86 \% y $97 \%$ de la longitud del brazo (Tabla I.)

El MTB fue inervado en la totalidad de la muestra por el NR, a través de ramos originados desde el nivel axilar hasta el tercio proximal del brazo. El NR emitió entre dos y cinco ramos motores, de los que en 24 casos $(50 \%)$ se presentaron cuatro ramos, mientras que en $12(25 \%)$ de los especímenes se observó tres ramos; el $25 \%$ restante presentó, dos (5 muestras) o cinco ramos motores (7 muestras). El primer ramo inervó la cabeza larga en el $100 \%$ de los casos y emergió en 44 muestras (92\%) a 35,6 DE 13,5 mm proximal al margen lateral de la banda tendinosa de inserción del músculo latísimo del dorso, en tanto que en 4 casos $(8 \%)$ este ramo se originó a 16,1 DE 9,9 mm distal al punto de referencia (Fig. 2). La distribución de los ramos motores emergentes del NR se tipificó en 6 patrones posibles, de acuerdo con su número y forma; estos se describen en la Tabla II. Los patrones más prevalentes fueron el I $(45,8 \%)$ y el II $(22,9 \%)$, mientras que el IIB fue el menos frecuente $(2,08 \%)$.

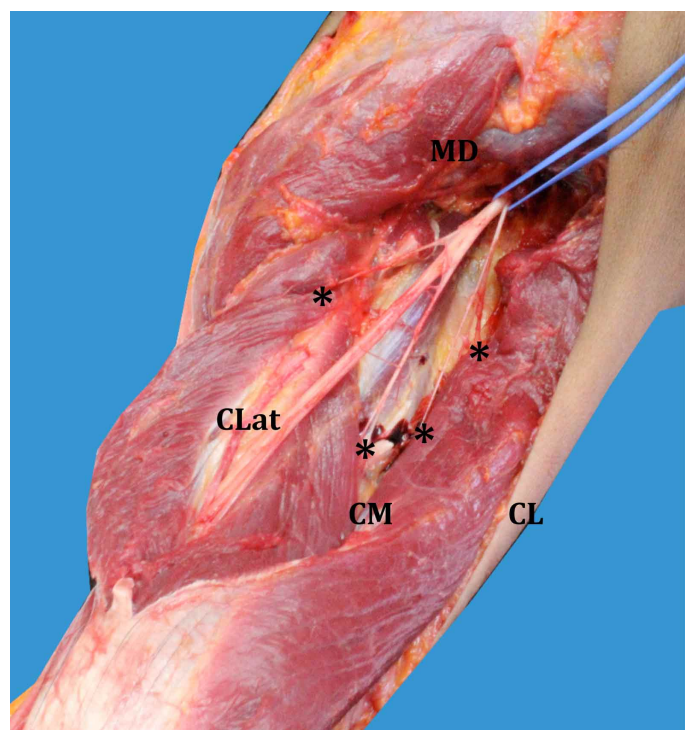

Fig. 2. Musculo tríceps braquial izquierdo. MD: músculo deltoides. CLat: cabeza lateral. CL: cabeza larga. CM: cabeza medial del tríceps braquial. $(*)$ puntos motores para la cabeza larga.

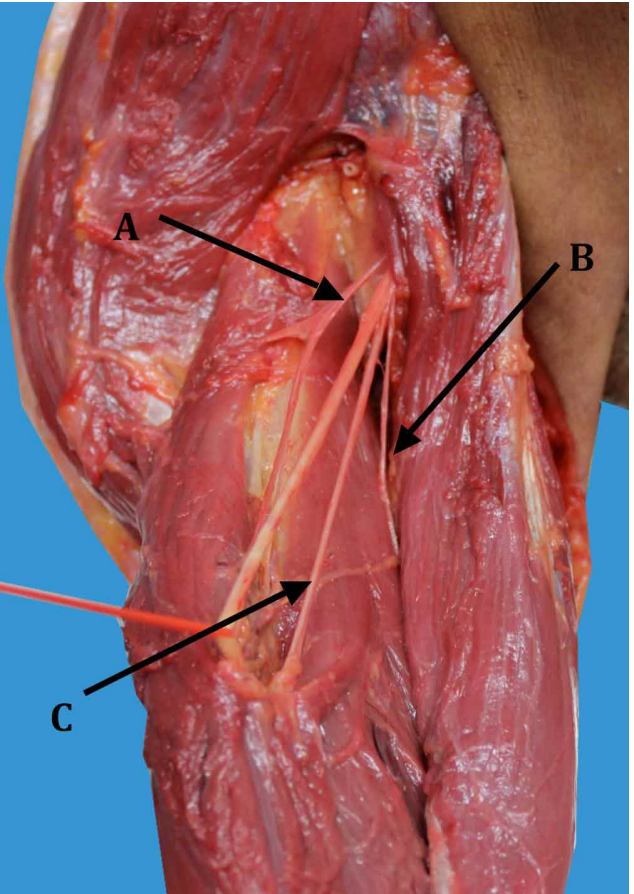

Fig. 3. Inervación del musculo tríceps braquial izquierdo. Distribución de los ramos motores del radial para las tres cabezas del tríceps braquial. Las letras señalan los ramos motores para cada vientre del tríceps braquial, así: A) cabeza lateral; B) cabeza larga; C) cabeza medial.

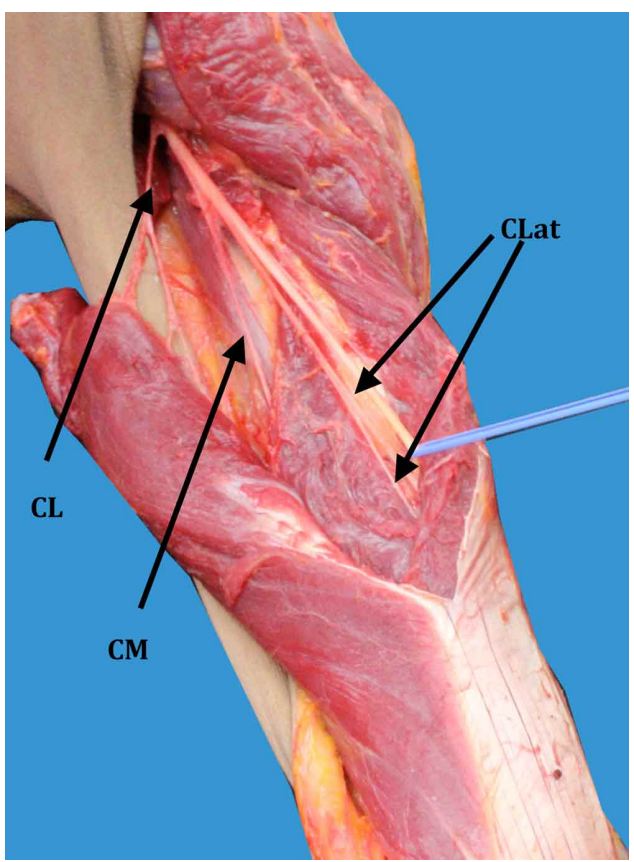

Fig. 4. Inervación del músculo tríceps braquial. Se observan 4 ramos originándose del nervio radial, uno para la cabeza larga (CL), uno para la cabeza medial (CM) y dos para la cabeza lateral (CLat), lo cual configura un patrón de ramificación tipo I. 
TORRES, E. A.; BALLESTEROS, L. E. \& FORERO, P. L. Biometría del músculo tríceps braquial y de sus puntos motores. Un estudio anatómico directo en una muestra de población Colombiana. Int. J. Morphol., 36(3):948-954, 2018.

Tabla I. Longitud de las cabezas del músculo tríceps braquial en 48 especímenes colombianos. Valores expresados en milímetros.

\begin{tabular}{|c|c|c|c|c|c|}
\hline & $\begin{array}{l}\text { Longitud cabeza } \\
\text { larga }\end{array}$ & $\begin{array}{l}\text { Longitud miotendinosa } \\
\text { proximal }\end{array}$ & $\begin{array}{l}\text { Longitud cabeza } \\
\text { medial }\end{array}$ & $\begin{array}{l}\text { Longitud cabeza } \\
\text { lateral }\end{array}$ & $\begin{array}{l}\text { Longitud miotendinosa } \\
\text { distal }\end{array}$ \\
\hline Media & 288,3 & 122,4 & 230 & 255,6 & 139,5 \\
\hline Mediana & 286,5 & 130 & 227 & 253 & 136 \\
\hline Desv. Estándar & 29,3 & 22,3 & 20,3 & 21,5 & 18,3 \\
\hline Mínimo & 208 & 52 & 179 & 195 & 105 \\
\hline Máximo & 338 & 156 & 266 & 289 & 184 \\
\hline
\end{tabular}

Tabla II. Patrones de distribución de los ramos motores del NR para el MTB en 48 especímenes colombianos.

\begin{tabular}{lll}
\hline Patrón & Prevalencia & Características \\
\hline IA & $45,8 \%$ & 4 ramos: dos para dos cabezas (uno respectivamente) y dos para la última de ellas) \\
I B & $4,16 \%$ & $\begin{array}{l}\text { 4 ramos: el primero se bifurca para la cabeza larga y la lateral, los dos siguientes para la cabeza la teral y el } \\
\text { último para la medial. }\end{array}$ \\
II A & $22,9 \%$ & 3 ramos, uno para cada vientre muscular. \\
II B & $2,08 \%$ & 3 ramos: uno para la cabeza larga, uno para la medial y el último se bifurca para la medial y lateral. \\
III & $14,5 \%$ & 5 ramos con diferente distribución para cada vientre. \\
IV & $10,4 \%$ & 2 ramos: uno independiente y el otro se bifurca para los otros dos vientres.
\end{tabular}

Se presentó gran variabilidad en la presencia de PM. La cabeza larga recibió entre uno y once, la medial entre uno y siete y la lateral entre dos y ocho PM. La cabeza larga recibió con mayor frecuencia tres PM (26,6\%), ubicados en el 85 $\%$ de los especímenes a $4-10 \mathrm{~cm}$ del origen muscular; la cabeza lateral recibe tres PM en el 44,4\% de la muestra, mientras que la cabeza medial recibió dos PM en 11 especímenes
(33,3\%), ubicados entre $4-10 \mathrm{~cm}$ de su origen (Tabla III). El número total de PM, varió entre ocho y 20, con un promedio de 11 DE 3 PM. No se encontraron diferencias estadísticamente significativas entre el número de PM del MTB izquierdo en relación al derecho $(\mathrm{p}=0,578)$. En la totalidad de las muestras evaluadas, los PM ingresan por la cara medial y al nivel del tercio proximal de los vientres musculares (Fig. 3).

Tabla III. Distribución de los puntos motores en en 48 especímenes colombianos. El área de dispersión toma como referencia el punto más alto del origen muscular.

\begin{tabular}{|c|c|c|c|c|}
\hline \multirow{2}{*}{ Vientre } & \multirow{2}{*}{$\begin{array}{l}\text { Número } \\
\text { de PM }\end{array}$} & \multicolumn{2}{|c|}{ Casos } & \multirow{2}{*}{$\begin{array}{c}\text { Área de } \\
\text { Dispersión }(\mathrm{mm})\end{array}$} \\
\hline & & $\mathrm{N}^{\mathrm{o}}$ & $\%$ & \\
\hline \multirow{10}{*}{ Cabeza larga } & 1 & 1 & 3 & \multirow{10}{*}{$35,64-136,26$} \\
\hline & 2 & 1 & 3 & \\
\hline & 3 & 8 & 26,6 & \\
\hline & 4 & 7 & 23,3 & \\
\hline & 5 & 5 & 16,6 & \\
\hline & 6 & 2 & 6,6 & \\
\hline & 7 & 2 & 6,6 & \\
\hline & 8 & 2 & 6,6 & \\
\hline & 10 & 1 & 3 & \\
\hline & 11 & 1 & 3 & \\
\hline \multirow{5}{*}{ Cabeza lateral } & 2 & 5 & 15 & \multirow{5}{*}{$22,98-130,12$} \\
\hline & 3 & 15 & 44,4 & \\
\hline & 4 & 6 & 18 & \\
\hline & 5 & 6 & 18 & \\
\hline & 8 & 1 & 3 & \\
\hline \multirow{7}{*}{ Cabeza medial } & 1 & 1 & 3 & \multirow{7}{*}{$15,83-154,19$} \\
\hline & 2 & 11 & 33,3 & \\
\hline & 3 & 11 & 33,3 & \\
\hline & 4 & 3 & 9 & \\
\hline & 5 & 1 & 3 & \\
\hline & 6 & 5 & 15 & \\
\hline & 7 & 1 & 3 & \\
\hline
\end{tabular}




\section{DISCUSIÓN}

En el presente estudio los datos de morfometría y de inervación del MTB derecho no presenta diferencias significativas con relación al izquierdo. En muchas de las variables estudiadas no fue posible establecer comparaciones entre nuestros hallazgos y lo descrito en estudios previos, en razón a que estos no reportan las características morfométricas de estas estructuras, por lo que los datos obtenidos en el presente trabajo adquieren relevancia en el ejercicio docente y pueden ser contrastados en nuevos estudios que se realicen sobre este tema.

En concordancia con estudios previos no encontramos especímenes con cuarta cabeza del MTB; esta ha sido reportada ocasionalmente (Fabrizio \& Clemente; Tubbs et al.; Cheema \& Singla; Nanjundaiah et al., 2012; Sawant; Erhardt \& Futterman, 2017). Se destaca el estudio de Sawant que reporta esta variación en el $2 \%$, con un vientre muscular inervado por el NR y originada "en la superficie anteromedial de la diáfisis humeral, justo por debajo del cuello quirúrgico" (Sawant; Nayak et al.). Una cuarta cabeza de este músculo puede estar relacionada con una mayor capacidad de generación de torque en extensión del antebrazo; sin embargo, la existencia de este componente muscular supernumerario puede favorecer el desarrollo de síndromes compresivos sobre el NR, que pueden afectar la extensión de antebrazo, muñeca y dedos.

La morfometría de los segmentos miotendinosos del tríceps no ha sido reportada en la literatura. En este trabajo se encontró que la longitud miotendinosa proximal representó el 42,5\% de la longitud total del músculo, mientras que la miotendinosa distal representó el 48,1 \% del mismo. En los procesos de reparación y cicatrización muscular, la reparación del tejido muscular magro tiene niveles y procesos diferentes a los de las porciones en las que se ubica tejido tendinoso de recubrimiento. Los modelos de estudio de reparación del músculo enfatizan en la capacidad de recuperación de las fibras musculares, sin considerar la diferencia estructural y funcional de los segmentos miotendinosos propios del tríceps que puede generar un residuo cicatrizal que interfiere funcionalmente en el músculo post lesión, por lo que la variable de segmentos miotendinosos presentes en el MTB debería ser tenida en cuenta en estudios que se ocupen de este tema (Lieber et al., 1992; Lagrota-Candido et al., 2010).

En concordancia con lo descrito mayoritariamente en la literatura, en la presente serie se observó la inervación de las tres cabezas del MTB procedente de ramos del NR. Algunos reportes de caso han descrito la inervación del MTB, específicamente de su cabeza larga, por ramas del nervio axilar (Nanjundaiah et al.). En este campo destaca el estudio de de Sèze et al. (2004) realizado en cadáveres embalsamados y pacientes sometidos a procedimiento quirúrgico de plexo braquial, que reporta inervación parcial de la cabeza larga del MTB por parte del nervio axilar en el 68,5\%. Un estudio más reciente en población latinoamericana encontró ramos del nervio axilar inervando la cabeza larga del MTB en el $100 \%$ de la muestra (12 piezas); el tejido identificado como ramo motor fue analizado histológicamente por medio de tinción de Hematoxilina-Eosina, confirmando la presencia de tejido nervioso (Molina et al., 2017).

Las diferencias entre los resultados del presente estudio y lo reportado por de Sèze $e t$ al. pueden atribuirse a las diferencias de abordaje. El estudio en mención realizó una disección desde la parte anterior de cuello y brazo y disecó las estructuras del plexo braquial de proximal a distal, lo cual generaba dificultad para identificar plenamente los ramos nerviosos procedentes del NR y del nervio axilar. En el caso del estudio de Molina et al. el abordaje de disección fue muy similar al del presente estudio. Estas divergencias en los hallazgos, exige evaluar la inervación complementaria del MTB en futuros proyectos de investigación. En la muestra estudiada, el patrón de inervación de las cabezas del MTB más prevalente fue el número uno (Fig. 4); sin embargo, es necesario promover estudios adicionales y desde múltiples abordajes para establecer un análisis más amplio al respecto.

La información sobre la distribución de las ramas del NR hacia el MTB señalada en este estudio además de enriquecer el conocimiento sobre esta estructura es relevante para el diseño de transferencias nerviosas hacia músculos lesionados, que permite retirar uno de los ramos para proveer inervación al músculo deltoides, por ejemplo, sin alterar la función de extensión de antebrazo al mantenerse indemne la inervación de las dos cabezas musculares restante. El punto de referencia de elección para estos procedimientos es el margen inferior de la inserción del latísimo del dorso, tal como fue utilizado en el presente estudio, o el margen inferior del músculo redondo mayor (Carlan et al., 2007; Uerpairojkit et al.).

El punto de origen del ramo para cada vientre muscular del MTB puede ser determinante al elegir un ramo donante en la recuperación de la función del músculo deltoides, en los casos de lesión alta del plexo braquial. Otros factores a considerar son el número de ramos motores que recibe cada vientre muscular y el aporte que cada cabeza realiza a la extensión del codo. Dado que la cabeza lateral recibe usualmente un solo ramo motor, algunos autores han señalado que es preferible utilizar alguno de los ramos que 
inervan a la cabeza medial (Tung \& Mackinnon, 2010). Sin embargo, considerando que el aporte de la cabeza larga a la extensión del codo resulta ser la menos significativa en comparación con los otros dos vientres y que el origen del ramo para la cabeza larga es el más proximal en comparación con los demás ramos motores, se recomienda el uso de este ramo en transferencia nerviosa (Ozer et al., 2006; Bertelli et al.; Uerpairojkit et al.; Al-Meshal \& Gilbert).

En el presente estudio se observó entre ocho y 20 PM para el MTB siendo la cabeza larga la que recibió mayor cantidad de PM con diferencia estadísticamente significativa con relación a los PM para las cabezas lateral y medial $(\mathrm{P}=0,005 ; 0,001$ respectivamente). No existen estudios previos que permitan contrastar esta información. Este estudio revela que sólo para la cabeza lateral se presenta una posible correlación entre la longitud muscular y el número de PM, lo que indica que a mayor longitud de la cabeza muscular existen mayor número de PM. En las demás cabezas del MTB no se evidenció esta correlación, posiblemente a que este estudio se limitó a la descripción de los PM, sin evaluar el recorrido nervioso intrafascicular, escenario en donde podrían surgir varios PM que permitiría evidenciar la correlación entre longitud del musculo y número de PM.

El área de dispersión de los PM resulta un dato de relevancia en el contexto clínico. La ubicación de los PM es esencial para el adecuado uso de terapia de estimulación eléctrica. La aplicación de corriente eléctrica de alto voltaje es utilizada como modalidad física de rehabilitación en lesiones nerviosas, musculares o neuromusculares en las que existe compromiso primario de la función motora, por lo que se resalta la necesidad de localizar los PM con el fin de optimizar los tratamientos. En este sentido, los resultados presentados en el presente estudio se constituyen en excelente guía que facilita realizar estas acciones terapéuticas (Botter et al., 2011; Gobbo et al., 2011, 2014; Okuma et al.).

TORRES, E. A.; BALLESTEROS, L. E. \& FORERO, P. L. Biometry of the brachial triceps muscle and its motor points. a direct anatomical study in a sample of Colombian population. Int J. Morphol., 36(3):948-954, 2018.

SUMMARY: The anatomical characteristics of the triceps brachii (TBM) muscle determine the repair speed and torque generation capacity. The TBM is typically innervated by the radial nerve $(\mathrm{RN})$, but there are reports of innervation by the axillary nerve. The aim of this study was to evaluate musculotendinous and nervous components of TBM. Through direct dissection in a sample of 48 arms of the Colombian mestizo population, we evaluated musculotendinous morphometry, nerve branches, motor points (MP) and origin of TBM innervation. The lengths of the medial, lateral and long heads of the TBM corresponded to 77.3
$\%, 86 \%$ and $97 \%$ of the arm length respectively. The TBM was innervated in the whole sample by the NR who issued between two and five motor branches. The first branch innervated the long head in $100 \%$ of cases and emerged laterally to the tendinous band insertion of the latissimus dorsi muscle. The long head received three MP $(26.6 \%)$, located in $85 \%$ of the specimens at $4-10 \mathrm{~cm}$ of muscular origin; while the lateral head received three MP in $44.4 \%$ of the sample. There were no statistically significant differences between the number of MP of the left TBM in relation to the right ( $p=0.578)$. The dispersion area of MP, as well as TBM morphometry are clinically relevant due to their use in neuromuscular electrical stimulation and nerve transfers. The findings presented in this study are a guide to facilitate results in this type of therapeutic action.

KEY WORDS: Triceps brachii muscle; Motor points; Morphometry; Radial nerve.

\section{REFERENCIAS BIBLIOGRÁFICAS}

Al-Meshal, O. \& Gilbert, A. Triceps innervation pattern: Implications for triceps nerve to deltoid nerve transfer. BioMed Res. Int., 2013:132954, 2013.

Bertelli, J. A.; Santos, M. A.; Kechele, P. R.; Ghizoni, M. F. \& Duarte, H. Triceps motor nerve branches as a donor or receiver in nerve transfers. Neurosurgery, 61(5 Suppl.):333-8, 2007.

Botter, A.; Oprandi, G.; Lanfranco, F.; Allasia, S.; Maffiuletti, N. A. \& Minetto, M. A. Atlas of the muscle motor points for the lower limb: implications for electrical stimulation procedures and electrode positioning. Eur. J. Appl. Physiol., 111(10):2461-71, 2011.

Carlan, D.; Pratt, J.; Patterson, J. M.; Weiland, A. J.; Boyer, M. I. \& Gelberman, R. H. The radial nerve in the brachium: an anatomic study in human cadavers. J. Hand Surg. Am., 32(8):1177-82, 2007.

Carter, R.; Lubinsky, J. \& Domholdt, E. Rehabilitation Research. Principles and Applications. $4^{\text {th }}$ ed. St Louis Mo., Elsevier Saunders, 2011. pp.22944.

Cheema, P. \& Singla, R. Four headed triceps brachii muscle. Int. J. Anat. Var., 4:43-4, 2011.

de Sèze, M. P.; Rezzouk, J.; de Sèze, M.; Uzel, M.; Lavignolle, B.; Midy, D. \& Durandeau, A. Does the motor branch of the long head of the triceps brachii arise from the radial nerve? An anatomic and electromyographic study. Surg. Radiol. Anat., 26(6):459-61, 2004.

Erhardt, A. J. \& Futterman, B. Variations in the Innervation of the Long head of the triceps brachii: a cadaveric investigation. Clin. Orthop. Relat. Res., 475(1):247-50, 2017.

Fabrizio, P. A. \& Clemente, F. R. Variation in the triceps brachii muscle: a fourth muscular head. Clin. Anat., 10(4):259-63, 1997.

Gobbo, M.; Gaffurini, P.; Bissolotti, L.; Esposito, F. \& Orizio, C. Transcutaneous neuromuscular electrical stimulation: influence of electrode positioning and stimulus amplitude settings on muscle response. Eur J. Appl. Physiol., 111(10):2451-9, 2011.

Gobbo, M.; Maffiuletti, N. A.; Orizio, C. \& Minetto, M. A. Muscle motor point identification is essential for optimizing neuromuscular electrical stimulation use. J. Neuroeng. Rehabil., 11:17, 2014.

Lagrota-Candido, J.; Canella, I.; Pinheiro, D. F.; Santos-Silva, L. P.; Ferreira, R. S.; Guimarães-Joca, F. J.; Lannes-Vieira, J. \& Quirico-Santos, T. Characteristic pattern of skeletal muscle remodelling in different mouse strains. Int. J. Exp. Pathol., 91(6):522-9, 2010.

Lee, N. G.; You, J. H.; Park, H. D.; Myoung, H. S.; Lee, S. E.; Hwang, J. H.; Kim, H. S.; Kim, S. S. \& Lee, K. J. The validity and reliability of 
TORRES, E. A.; BALLESTEROS, L. E. \& FORERO, P. L. Biometría del músculo tríceps braquial y de sus puntos motores. Un estudio anatómico directo en una muestra de población Colombiana. Int. J. Morphol., 36(3):948-954, 2018.

the motor point detection system: a preliminary investigation of motor points of the triceps surae muscles. Arch. Phys. Med. Rehabil., 90(2):348-53, 2009

Lieber, R. L.; Jacobson, M. D.; Fazeli, B. M.; Abrams, R. A. \& Botte, M. J. Architecture of selected muscles of the arm and forearm: anatomy and implications for tendon transfer. J. Hand Surg. Am., 17(5):787-98, 1992.

Molina, C. R.; Díaz, I.; Taunton, M. J.; Flores, E.; Rosas, C. \& Letelier, R. Innervation of the long head of triceps brachii muscle. Int. J. Morphol., 35(2):442-4, 2017.

Nanjundaiah, K.; Jayadevaiah, S. M. \& Chowdapurkar, S. Long head of tríceps supplied by axillary nerve. Int. J. Anat. Var., 5:35-7, 2012.

Nayak, S. R.; Krishnamurthy, A.; Prabhu, L. V.; Rai, R.; Ranade, A. V. \& Madhyastha, S. Anatomical variation of radial wrist extensor muscles: a study in cadavers. Clinics (Sao Paulo), 63(1):85-90, 2008

Okuma, Y.; Bergquist, A. J.; Hong, M.; Chan, K. M. \& Collins, D. F. Electrical stimulation site influences the spatial distribution of motor units recruited in tibialis anterior. Clin. Neurophysiol., 124(11):225763, 2013.

Ozer, H.; Açar, H. I.; Cömert, A.; Tekdemir, I.; Elhan, A. \& Turanli, S. Course of the innervation supply of medial head of triceps muscle and anconeus muscle at the posterior aspect of humerus (anatomical study). Arch. Orthop. Trauma Surg., 126(8):549-53, 2006.

Sawant, S. Study of variant heads of triceps muscle with its developmental basis. Int. J. Anal. Pharm. Biomed. Sci., (2):23-6, 2013.

Tubbs, R. S.; Salter, E. G. \& Oakes, W. J. Triceps brachii muscle demonstrating a fourth head. Clin. Anat., 19(7):657-60, 2006.

Tung, T. H. \& Mackinnon, S. E. Nerve transfers: indications, techniques, and outcomes. J. Hand Surg. Am., 35(2):332-41, 2010.

Uerpairojkit, C.; Ketwongwiriya, S.; Leechavengvongs, S.; Malungpaishrope, K.; Witoonchart, K.; Mekrungcharas, N.; Chareonwat, B. \& Ongsiriporn, M. Surgical anatomy of the radial nerve branches to triceps muscle. Clin. Anat., 26(3):386-91, 2013.

Wang, L. H. \& Weiss, M. D. Anatomical, clinical, and electrodiagnostic features of radial neuropathies. Phys. Med. Rehabil. Clin. N. Am., 24(1):33-47, 2013.

Windisch, G.; Tesch, N. P.; Grechenig, W. \& Peicha, G. The triceps brachii muscle and its insertion on the olecranon. Med. Sci. Monit., 12(8):BR290-4, 2006.

\author{
Dirección para correspondencia: \\ Edgar Adrián Torres Sepúlveda, MSc, Prof. \\ Programa de Medicina \\ Línea de Ciencias Básicas Médicas \\ Facultad de Salud \\ Universidad Autónoma de Bucaramanga \\ Campus el Bosque \\ Calle 157 \#14-55 \\ Floridablanca - Santander \\ COLOMBIA
}

E-mail: etorres262@unab.edu.co

Recibido : 24-01-2018

Aceptado: 12-04-2018 